\title{
A NOTE ON TWIST SPUN KNOTS
}

\author{
JOSÉ MARÍA MONTESINOS ${ }^{1}$
}

ABSTRACT. A movie presentation for the twist spun knots of an arc is given.

Some time ago Francisco González-Acuña asked me for a movie presentation of the twist spun knots defined and studied by Zeeman [Z]. Since then, other low dimensional topologists have posed the same question to me. Perhaps the following easy solution may have some interest.

LEMMA. Let $K$ be the knot in plat presentation of Figure 1 where $x$ belongs to the braid group $B_{2 m+1}$. Then the $n$-twist spun knot of $K$ is given by the diagram of Figure 2.

REMARK. In Figure 2 we use Lomonaco's notation [L]. The diagram of Figure $3(\mathrm{a})$ is explained in Figure $3(\mathrm{~b})$. The critical saddle point occurs at level $t=\theta$.

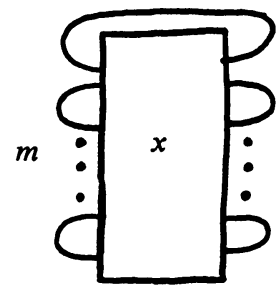

FIGURE 1.

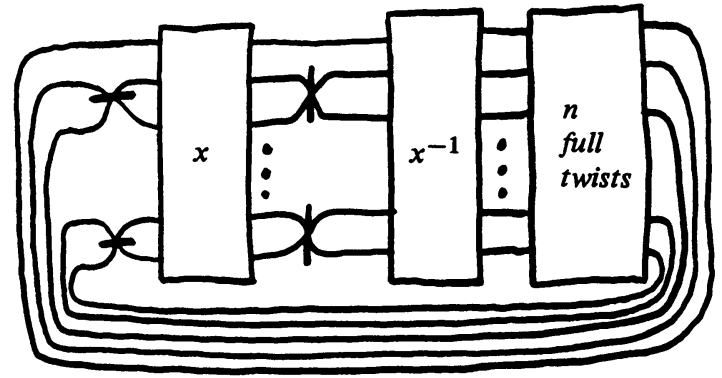

FIGURE 2.

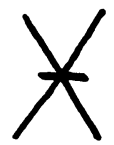

(a)

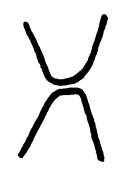

$\theta-\epsilon$

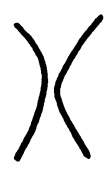

$\theta+\epsilon$

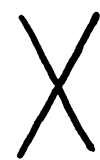

$\theta$

(b)

FIGURE 3.

Received by the editors June 11, 1985.

1980 Mathematics Subject Classification. Primary 57Q45; Secondary 57M25.

Key words and phrases. Twist spun knot, plat, braid, slice knot.

${ }^{1}$ Research supported in part by "Comite Conjunto Hispano-Norteamericano" and NSF Grant 8120790 . 

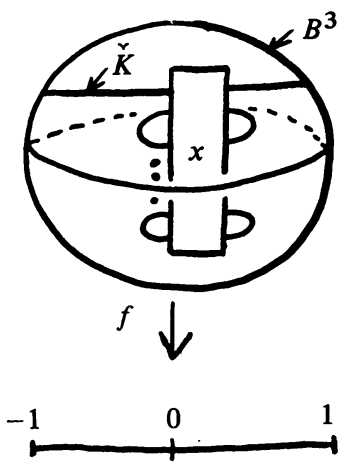

FIGURE 4.

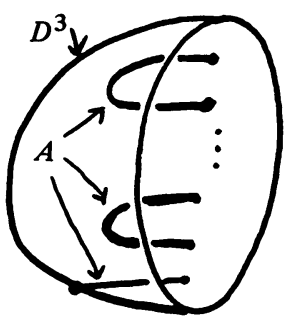

(a)

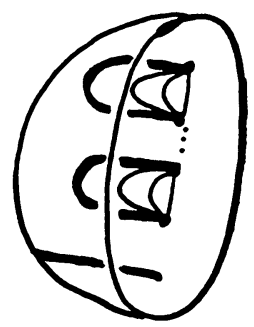

(b)

FigURE 5.

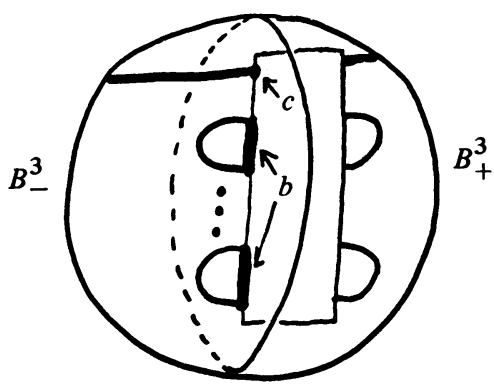

FigURE 6.

PROOF. By deleting the interior of a regular neighborhood of a point in $K$ we obtain a pair $\left(B^{3}, B^{3} \cap K\right)$ which we denote by $\left(B^{3}, \breve{K}\right)$. Consider the height function $f: B^{3} \rightarrow[-1,1]$ of Figure 4 .

Let $t \in[0,1]$ be the parameter measuring the spinning process of $B^{3}$ so that at time $t=1$ the ball arrives to its original position. Assume that the twisting of $\breve{K}$ occurs during the interval $\left[\frac{1}{2}, 1\right]$.

We want a movie of the $n$-twist spun knot $K_{n}$ of $K$ with respect to "hyperplane" sections $S_{r}^{3}, r \in(-1,1)$, where $S_{r}^{3}$ is the result of spinning the subset $f^{-1}(r)$ of $B^{3}$. For $r \in\{-1,1\}, f^{-1}(r)$ is just a point.

To achieve this we first define an isotopy of $S^{4}$ which places the saddle points of $(f \times \mathrm{id}) \mid K_{n}$ in the level $S_{0}^{3}$. This isotopy is defined in three steps.

Step 1. Consider the model halfball $D^{3}$ and the set $A$ of $m+1 \operatorname{arcs}$ shown in Figure 5(a). There is an isotopy $g: A \times I \rightarrow D^{3}$ which pushes $m \operatorname{arcs}$ of $A$ onto the boundary. In Figure 5(b) we see the images of $A$ for some values of the parameter. 


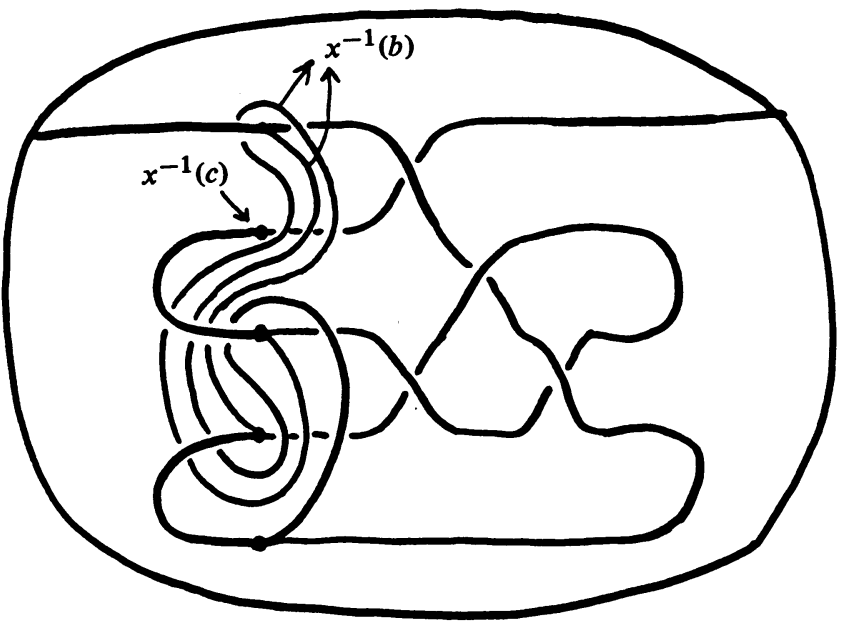

FIGURE 7.

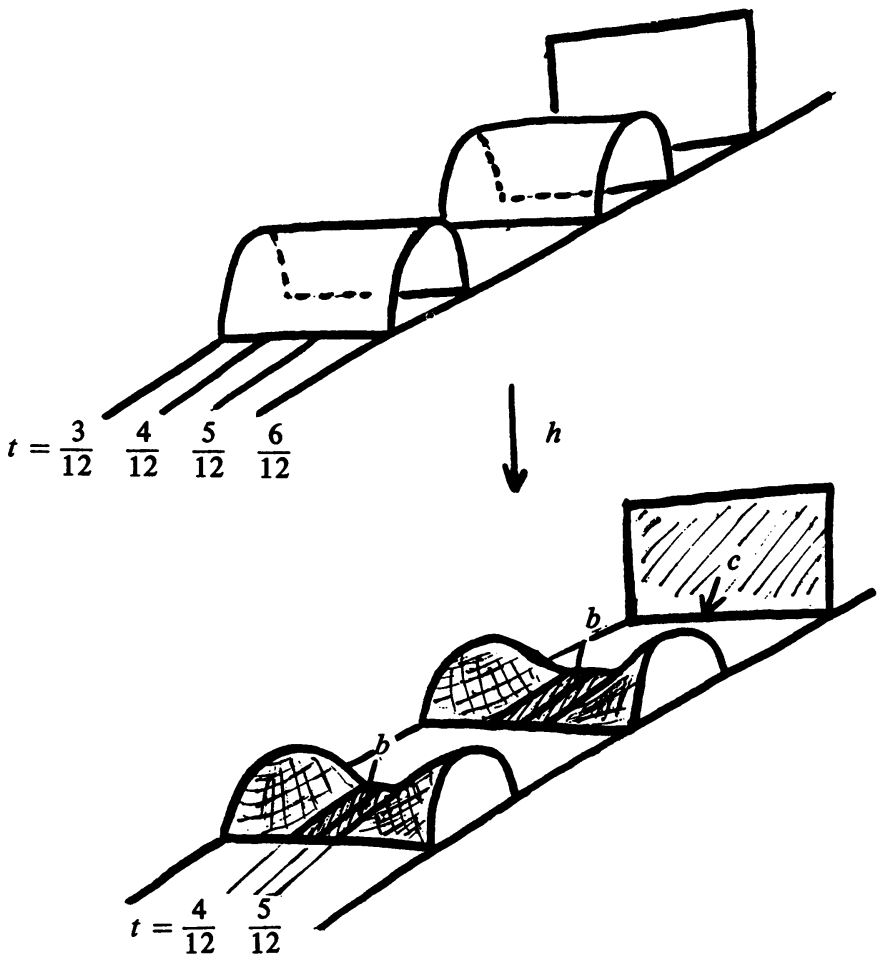

Figure 8.

Step 2. Let $B_{+}^{3}$ and $B_{-}^{3}$ be the halfballs $f^{-1}[0,1]$ and $f^{-1}[0,-1]$ of $B^{3}$. Let $F_{+}$and $F_{-}$be homeomorphisms $F_{ \pm}:\left(D^{3}, A\right) \rightarrow\left(B_{ \pm}^{3}, B_{ \pm}^{3} \cap \breve{K}\right)$ and define isotopies $g_{ \pm}: \check{K} \times I \rightarrow B^{3}$ as follows: $g_{ \pm}$is the identity map in $\left(B_{\mp}^{3} \cap \check{K}\right) \times t, t \in I$, and equals $F_{ \pm} g F_{ \pm}^{-1}$ in $\left(B_{ \pm}^{3} \cap \check{K}\right) \times I$. We embed $g_{ \pm}$in ambient isotopies $G_{ \pm}: B^{3} \times I \rightarrow B^{3}$. Note that $G_{-}\left(\left(B_{-}^{3} \cap \breve{K}\right) \times 1\right)$ is the set of $\operatorname{arcs} b$ together with the point $c$ of Figure 6 , if we think of $F_{-}$as the identity map. Under this condition the set $G_{+}\left(\left(B_{+}^{3} \cap \breve{K}\right) \times 1\right)$ 


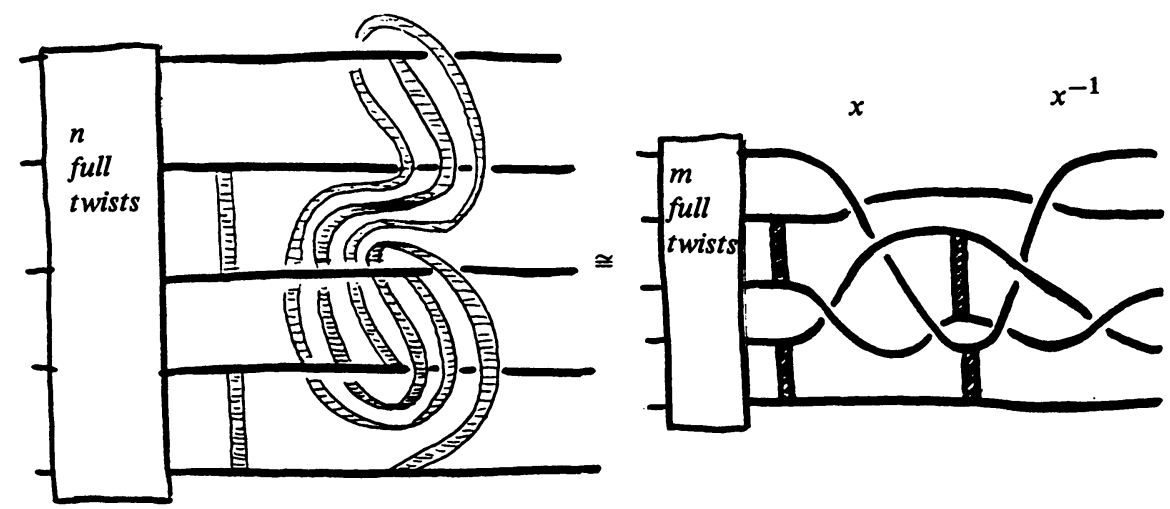

FIGURE 9.

is the image of $b \cup c$ under the action of $x^{-1} \in B_{2 m+1}$ on $\left(f^{-1}(0), f^{-1}(0) \cap \breve{K}\right)$. In Figure 7 we show the case $x=\sigma_{2}^{-1} \sigma_{4}^{-1} \sigma_{3} \sigma_{2}^{-1}$.

Step 3. We now define an isotopy of $S^{4}$. This isotopy connects the identity map with a map $h: S^{4} \rightarrow S^{4}$ defined as follows. The map $h$ realizes $G_{+}$when we spin $B^{3}$ between $t=0$ and $t=1 / 12$, it is constant for $t \in\left[\frac{1}{12}, \frac{2}{12}\right]$, and undoes $G_{+}$ between $t=2 / 12$ and $t=3 / 12$. After that, $h$ does $G_{-}$in $\left[\frac{3}{12}, \frac{4}{12}\right]$, is constant in $\left[\frac{4}{12}, \frac{5}{12}\right]$ and undoes $G_{-}$in $\left[\frac{5}{12}, \frac{6}{12}\right]$. During $\left[\frac{1}{2}, 1\right] h$ is the identity map. In Figure 8 we see $h\left(\left(B_{-}^{3} \cap \breve{K}\right) \times\left[\frac{3}{12}, \frac{6}{12}\right]\right)$.

The knot $h\left(K_{n}\right)$ is ambient isotopic to $K_{n}$ but all its saddle points with respect to $f \times$ id are at level $S_{0}^{3}$. We only need to understant $S_{0}^{3} \cap h\left(K_{n}\right)$. The pair $\left(S_{0}^{3}, S_{0}^{3} \cap\right.$ $\left.h\left(K_{n}\right)\right)$ is the union of the result of spinning $\left(f^{-1}(0), f^{-1}(0) \cap \breve{K}\right)$ during $t \in\left[0, \frac{1}{2}\right]$, with the result of spinning $x^{-1}(b \cup c)$ during $t \in\left[\frac{1}{12}, \frac{2}{12}\right]$, with the result of spinning $b \cup c$ during $t \in\left[\frac{4}{12}, \frac{5}{12}\right]$, with the result of $n$-twist spinning $\left(f^{-1}(0), f^{-1}(0) \cap \breve{K}\right)$ during $t \in\left[\frac{1}{2}, 1\right]$. The picture for $\breve{K}$ given by $x=\sigma_{2}^{-1} \sigma_{4}^{-1} \sigma_{3} \sigma_{2}^{-1}$ is in Figure 9.

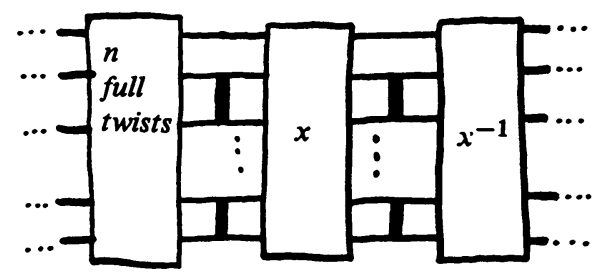

FIGURE 10.

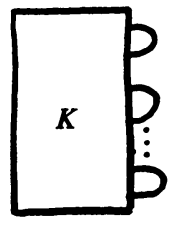

(a) $K$ is a knot

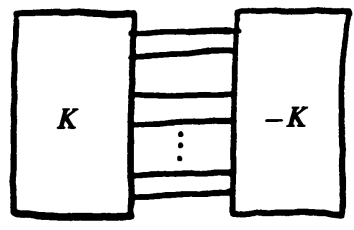

(b) $-K$ is the mirror image

Figure 11. 
We have that $\left(S_{0}^{3}, S_{0}^{3} \cap h\left(K_{n}\right)\right)$ is a torus link with $2 m+1$ trivial components and $n$-full twists, together with two sets of bands which correspond to the saddle points. By shrinking the bands with middle lines $x^{-1}(b \cup c)$ suitably we see that Figure 9 becomes Figure 10.

COROLlaRY. The torus link $\{(2 m+1) n, 2 m+1\}$ is a slice of a trivial knot in $S^{4}$. Links of the form depicted in Figure 11(b) have the same property.

PROOF. For the first part take $x \in B_{2 m+1}$ such that $K$ is a trivial knot. For the second part, remember that the 1-twist spun knot of $K$ is trivial [Z].

\section{REFERENCES}

[F] R. H. Fox, A quick trip through knot theory, Topology of 3-Manifolds and Related Topics (M. K. Fort, Jr., ed.), Prentice-Hall, Englewood Cliffs, N. J., 1962, pp. 110-167.

[L] S. J. Lomonaco, Jr., The homotopy groups of knots. I. How to compute the algebraic 2-type, Pacific J. Math. 95 (1981), 349-390.

[Z] E. C. Zeeman, Twisting spun knots, Trans. Amer. Math. Soc. 115 (1965), 471-495.

(a) $K$ is a knot

(b) $-K$ is the mirror image

Mathematical Sciences Research Institute, 1000 Centennial DRive, BerkeLEY, CALIFORNIA 94720

Current address: Facultad de Matemáticas, Universidad de Zaragoza, 50009 Zaragoza, Spain 